\title{
Statistical Linearization of Nonlinear Stiffness in Hydropneumatic Suspension
}

\author{
Zhongli Cheng ${ }^{1}$, Zonghua $\mathrm{Li}^{1}$ and Fanqing Kong ${ }^{1}$ \\ School of Mechanical Engineering, Beijing Institute of Technology Beijing, 100081, China
}

\begin{abstract}
Hydropneumatic springs are the elastic components of a vehicle's suspension. As the nonlinear characteristic of the spring is difficult to express accurately, the statistical linearization method is introduced to analyze the dynamic response of the hydropneumatic spring. The nonlinear stiffness of a hydropneumatic spring is approximated by a quadratic polynomial at the static equilibrium position. Parameters of the hydropneumatic spring, road roughness and vehicle velocity are provided and analytical functions for equivalent stiffness and the dynamic equilibrium position are worked out in this paper. The analytical functions are validated through numerical simulation and are shown to be more accurate than those validated by existing methods. The method proposed here could be used in the design and analysis of hydropneumatic suspensions in future.
\end{abstract}

\section{Introduction}

Hydropneumatic suspensions use pressed gas as the elastic medium, which exhibits the characteristic of nonlinear gradient stiffness. This provides better comfort and cross country ability so hydropneumatic suspensions are widely used in high performance cross country vehicles, military vehicles and mine trucks [1-3]. As its nonlinear characteristics restrict the application of linear system analysis, nonlinear modeling and simulation are adopted to analyse this suspension by MA, et al [4], FENG, et al [5] and useful conclusions are reached. However, explicit analytical formulae are still needed for complex hydropneumatic suspensions in engineering applications, especially at the design stage.

Vehicle suspensions can be modelled by a MDOF (multiple degree of freedom) nonlinear vibration system with wide-band random excitation, suitable for application of exact feedback linearization or statistical linearization [6-8]. Exact feedback linearization is adopted to analyze hydropneumatic suspension by LI, et al [9] and SHI, et al [10] and satisfactory results are achieved in simulation. But, exact feedback linearization has many drawbacks. It requires that all states be measurable and robustness cannot be guaranteed, so the method of statistical linearization of the nonlinear characteristic is still required. Statistical linearization is proposed by CAUGHEY, et al [11] and KAZAKOV, et al [12] independently and extended to MDOF systems by IWAN, et al [13]. ATALIK, et al [14-15] uses deformation of elastic elements to establish system differential equations so that statistical linearization of the system can be reduced to linearization of the elastic elements to simplify calculations. GAI, et al [16] concludes that the displacement of the sprung mass is a non-zero mean process in response to a zero mean unit white noise excitation because of the asymmetry of the stiffness. YOUSEFI, et al [17] and LOHMANN [18] apply a low-order approximation statistical linearization method to the nonlinear model of hydropneumatic suspension. CAO, et al [19] uses a finite Taylor series instead of the exponential function, but since only the first two terms of the series are considered, the asymmetry of the stiffness is typically neglected and this will cause considerable errors under conditions of large suspension deformation.

Based on the references mentioned above, a new method is proposed in this paper, which considers random road roughness as the excitation and a quadratic polynomial is introduced to take the asymmetry of the stiffness of a hydropneumatic suspension into consideration. The coordinates of the differential equations are composed of the deformations of the elastic element and the absolute displacement of the sprung mass. The explicit forms of the equivalent stiffness and the dynamic mean-value offset from the static equilibrium position are determined using the statistical linearization method. Lastly, the proposed model is validated through numerical simulation.

\section{Nonlinear Suspension Model Development}

2.1 Nonlinear elasticity of hydropneumatic
suspension


The mono chamber hydropneumatic spring is shown in Figure 1. Using the ideal gas equation, the gas pressure at any position can be expressed as:

$$
p_{0}\left(A_{1} h_{0}\right)^{\gamma}=p\left(A_{1} h_{0}+A_{2} z_{1}\right)^{\gamma}
$$

where $\gamma$ is the gas polytropic exponent, $A_{1}$ is the crosssectional area of the air chamber, $A_{2}$ is the ring chamber area of the hydropneumatic spring and $h_{0}$ and $p_{0}$ are the gas column length and the pressure of the hydropneumatic spring at static equilibrium position respectively.

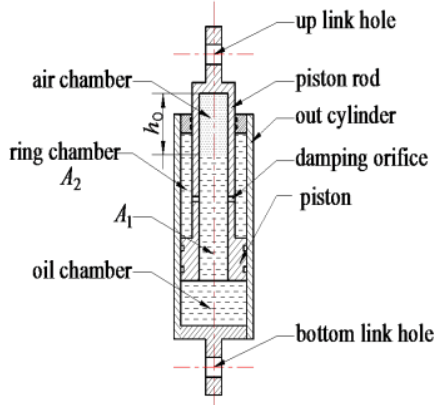

Figure 1. Cross-section of hydro-pneumatic spring

The rebound force of the hydropneumatic spring can be expressed as:

$$
F_{s}=\left(p-p_{0}\right) A_{1}=A_{1} p_{0}\left[\left(\frac{h_{0}}{h_{0}+\alpha z_{1}}\right)^{\gamma}-1\right]=W_{s}\left[\left(\frac{h_{0}}{h_{0}+\alpha z_{1}}\right)^{\gamma}-1\right]
$$

where $W_{s}$ is the weight of the sprung mass and $\alpha=A_{2}$ $/ A_{1}$ is the area ratio.

\subsection{Two-DOF hydropneumatic suspension model}

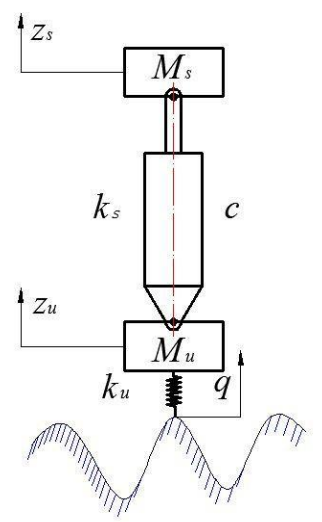

Figure 2. Model of 2-DOF hydropneumatic suspension

Ignoring the suspension links' effect and the damping of the tire, the differential equations of the system shown in Figure 2 are given in Eq. (3), assuming the damping of the hydropneumatic spring is linear.

$$
\left\{\begin{array}{c}
M_{s} \ddot{z}_{s}-F_{s}+c\left(\dot{z}_{s}-\dot{z}_{u}\right)=0 \\
M_{u} \ddot{z}_{u}+F_{s}-c\left(\dot{z}_{s}-\dot{z}_{u}\right)=k_{u}\left(q-z_{u}\right)
\end{array}\right.
$$

where

$M_{\mathrm{s}}$ and $M_{\mathrm{u}}$ : sprung and unsprung masses respectively; $k_{\mathrm{s}}$ : nonlinear stiffness of the hydropneumatic suspension;

$k_{\mathrm{u}}$ : tire stiffness;

$c$ : linear damping coefficient of the suspension;

$q$ : road roughness time-domain input;

$z_{\mathrm{s}}$ and $z_{\mathrm{u}}$ : the absolute displacements of $M_{\mathrm{s}}$ and $M_{\mathrm{u}}$.

According to references $[14,15]$, using the deformation of the spring $z_{1}=z_{\mathrm{s}}-z_{\mathrm{u}}$ and the absolute displacement of the sprung mass $z_{\mathrm{s}}$ as new general coordinates, Eq. (3) can be rewritten as:

$$
\left\{\begin{array}{c}
M_{s} \ddot{z}_{s}=F_{s}-c \dot{z}_{1} \\
M_{u}\left(\ddot{z}_{s}-\ddot{z}_{1}\right)-c \dot{z}_{1}=-F_{s}+c \dot{z}_{1}+k_{u}\left(q-z_{s}+z_{1}\right)
\end{array}\right.
$$

In Eq. (4), the nonlinear spring force is only related to the coordinate $z_{1}$, so statistical linearization of the system becomes the linearization of the nonlinear spring force. Substituting Eq. (2) into Eq. (4) gives:

$$
\left\{\begin{array}{c}
M_{s} \ddot{z}_{s}+W_{s}\left[\left(\frac{h_{0}}{h_{0}+\alpha z_{1}}\right)^{\gamma}-1\right]+c \dot{z}_{1}=0 \\
M_{u}\left(\ddot{z}_{s}-\ddot{z}_{1}\right)-W_{s}\left[\left(\frac{h_{0}}{h_{0}+\alpha z_{1}}\right)^{\gamma}-1\right]-c \dot{z}_{1}=k_{u}\left(q-z_{s}+z_{1}\right)
\end{array}\right.
$$

\section{Statistical Linearization of the 2-DOF Suspension System}

\subsection{Equivalent stiffness of the system}

Define a zero-mean variable $z_{10}$ as:

$$
z_{10}=z_{1}-E\left(z_{1}\right)=z_{1}-m_{z_{1}}
$$

The linearized spring force can be written as:

$$
F_{s}^{*}\left(z_{1}\right)=-k_{e q} z_{10}-F_{0}
$$

The difference between the nonlinear spring force and the linear-equivalent spring force is:

$$
\Delta F=F_{s}\left(z_{1}\right)-F_{s}^{*}\left(z_{1}\right)
$$

Using the minimization of $E\left(\Delta F^{2}\right)$ as the statistical linearization criteria gives:

$$
\frac{\partial E\left(\Delta F^{2}\right)}{\partial k_{e q}}=\frac{\partial E\left(\Delta F^{2}\right)}{\partial F_{0}}=0
$$

Reference [6] proves that the arrest point is the minimum value point. From Eq. (9), Eq. (10) and Eq. (11) can be determined:

$$
\begin{gathered}
F_{0}=E\left(F_{s}\right)=\int_{-\infty}^{+\infty} F_{s}\left(z_{1}\right) p\left(z_{1}\right) \mathrm{d} z_{1} \\
k_{e q}=\frac{E\left\{\left[z_{1}-E\left(z_{1}\right)\right] \cdot F_{s}\left(z_{1}\right)\right\}}{\sigma_{z_{1}}^{2}}=\frac{E\left[z_{10} \cdot F_{s}\left(z_{10}+m_{z 1}\right)\right]}{E\left(z_{10}^{2}\right)}
\end{gathered}
$$

Determining the mathematical expectation of the first equation in Eq. (3) gives:

$$
m_{s} \cdot E\left(\ddot{z}_{s}\right)-E\left(F_{s}\right)+c \cdot E\left(\dot{z}_{1}\right)=0
$$

Based on the assumption that ${ }^{\prime} z_{s}$ and ${ }^{\prime} z_{1}$ are both Gaussian processes, reference [2] gives: 


$$
E\left(\dot{z}_{s}\right)=E\left(\ddot{z}_{s}\right)=E\left(\dot{z}_{1}\right)=E\left(\ddot{z}_{1}\right)=0
$$

Substituting Eq. (13) into Eq. (10) gives:

$$
F_{0}=\int_{-\infty}^{+\infty} F_{s}\left(z_{1}\right) p\left(z_{1}\right) \mathrm{d} z_{1}=0
$$

Since $z_{1}$ is a stationary Gaussian random process, the probability density function of $z_{1}$ can be expressed as [20]:

$$
p\left(z_{1}\right)=\frac{1}{\sigma_{z 1} \sqrt{2 \pi}} \exp \left[-\frac{\left(z_{1}-m_{z_{1}}\right)^{2}}{2 \sigma_{z_{1}}^{2}}\right]
$$

From Eq. (11), $\mathrm{k}_{\mathrm{eq}}$ can be determined :

$$
k_{e q}=\frac{\int_{-\infty}^{+\infty}\left[z_{10} \cdot F_{s}\left(z_{1}\right) \cdot p\left(z_{1}\right)\right] \mathrm{d} z_{1}}{\sigma_{z_{1}}^{2}}
$$

\subsection{Quadratic polynomial of nonlinear spring force}

Since Eq. (16) can't be expressed in an explicit form, a quadratic polynomial of non-linear spring force $F_{s}\left(z_{l}\right)$ at the static equilibrium position is used to replace the exponential function.

$$
F_{s}\left(z_{1}\right)=\frac{1.478 W_{s} \alpha z_{1}}{h_{0}}-\frac{1.697 W_{s} \alpha^{2} z_{1}^{2}}{h_{0}^{2}}
$$

As shown in Eq. (17), the function of nonlinear spring force is composed of a polynomial, where the variable is $z_{1} / h_{0}$, (i.e. the ratio of the deflection of the hydropneumatic spring to the gas height of hydropneumatic spring at the static equilibrium position). In practical situations, it is required that the maximum absolute value of $z_{1}$ be smaller than one third of $h_{0}$ :

$$
\max \left|z_{1}\right| \leq \frac{1}{3} h_{0}
$$

Setting $z_{1}=h_{0} / 3$, gas polytropic exponent $\gamma=1.3$, it is noted that the nonlinear characteristic of the hydropneumatic spring cannot be approximated by the first term of a Taylor series as this will yield a maximum relative error of $\Delta F_{1}=20.74 \%$. An approximation using the first and third terms of a Taylor series will yield a larger error of $\Delta F_{1,3}=76.47 \%$ and it ignores the asymmetric characteristics of the hydropneumatic spring stiffness. An approximation using the first and second terms of a Taylor series, the even order terms of the expanding expression are certainly negative, and it exists error. An approximation using the first, second and third terms of a Taylor series, however, will enhance the accuracy of the calculation results with an increment in calculation complexity. Thus, a quadratic polynomial makes the calculations easier and accurate results can be obtained.

$z_{10}$ is a normal random process with zero mean, and the derivative of high level power exists. Reduced order rules are used with the high level power of normal distribution [14].

$$
\begin{gathered}
k_{e q}=W_{s}\left[\frac{1.478 \alpha}{h_{0}}-\frac{1.697 \alpha^{2}\left(m_{z 1}^{3}+m_{z 1} \sigma_{z 1}^{2}\right)}{\sigma_{z 1}^{2} h_{0}^{2}}\right] \\
F_{0}=\int_{-\infty}^{\infty}\left(\frac{1.478 W_{s} \alpha z_{1}}{h_{0}}-\frac{1.697 W_{s} \alpha^{2} z_{1}^{2}}{h_{0}^{2}}\right) p\left(z_{1}\right) d z_{1}= \\
\frac{1.478 W_{s} \alpha}{h_{0}} E\left(z_{1}\right)-\frac{1.697 W_{s} \alpha^{2}}{h_{0}^{2}} E\left(z_{1}^{2}\right)=0
\end{gathered}
$$

\subsection{Response of linear-equivalent suspension system}

In order to determine $k_{\mathrm{eq}}$, the mean value and the variance of $z_{1}$ are needed. Thus, the linear-equivalent system is introduced to determine the mean value and variance of $z_{1}$.

Define the following:

$$
z_{s 0}=z_{s}-E\left(z_{s}\right)=z_{s}-m_{z_{s}}
$$

Since $z_{\mathrm{s}}$ is a Gaussian process,

$$
\dot{z}_{s}=\dot{z}_{s 0}, \quad \ddot{z}_{s}=\ddot{z}_{s 0}
$$

Substituting Eq. (7), Eq. (21) and Eq. (22) into Eq. (4) gives:

$$
\left\{\begin{array}{c}
M_{s} \ddot{z}_{s 0}+c \dot{z}_{10}+k_{e q} z_{10}=0 \\
M_{u}\left(\ddot{z}_{s 0}-\ddot{z}_{10}\right)-c \dot{z}_{10}-\left(k_{e q}+k_{u}\right) z_{10}+k_{u} z_{s 0}=k_{u}\left(q-m_{z_{s}}+m_{z_{1}}\right)
\end{array}\right.
$$

Noting that the terms on the left-hand side and $q$ are all zero-mean processes, the following can be obtained by determining the mathematical expectation of the second equation in Eq. (23):

$$
-m_{z_{s}}+m_{z_{1}}=0
$$

So, Eq. (23) can be simplified:

$$
\left\{\begin{array}{c}
M_{s} \ddot{z}_{s 0}+c \dot{z}_{10}+k_{e q} z_{10}=0 \\
M_{u}\left(\ddot{z}_{s 0}-\ddot{z}_{10}\right)-c \dot{z}_{10}+k_{u} z_{s 0}-\left(k_{e q}+k_{u}\right) z_{10}=k_{u} q
\end{array}\right.
$$

The matrix form of Eq. (25) is as follows:

$$
\begin{array}{r}
{\left[\begin{array}{cc}
M_{s} & 0 \\
M_{u} & -M_{u}
\end{array}\right]\left\{\begin{array}{l}
z_{s 0} \\
z_{10}
\end{array}\right\}+\left[\begin{array}{cc}
0 & c \\
0 & -c
\end{array}\right]\left\{\begin{array}{l}
z_{s 0} \\
z_{10}
\end{array}\right\}+} \\
{\left[\begin{array}{cc}
0 & k_{e q} \\
k_{u} & -\left(k_{e q}+k_{u}\right)
\end{array}\right]\left\{\begin{array}{l}
z_{s 0} \\
z_{10}
\end{array}\right\}=k_{u}\left\{\begin{array}{l}
0 \\
q
\end{array}\right\}}
\end{array}
$$

The transfer function can then be worked out:

$$
\left\{\begin{array}{c}
H_{z_{s 0}-q} \\
H_{z_{10}-q}
\end{array}\right\}=\frac{1}{\Delta}\left\{\begin{array}{c}
k_{u}\left(k_{e q}-c j \omega\right) \\
-\omega^{2} M_{s} k_{u}
\end{array}\right\}
$$

Where

$$
\Delta=\omega^{2} M_{s}\left(k_{u}-k_{e q}+\mathrm{j} c \omega-\omega^{2} M_{u}\right)+\left(k_{u}-\omega^{2} M_{u}\right)\left(k_{e q}-\mathrm{j} c \omega\right)
$$

The excitation spectrum of a vehicle travel can be expressed as:

$$
G_{q 0}(\omega)=(2 \pi)^{2} G_{q}\left(n_{0}\right) n_{0}^{2} \frac{v}{\omega^{2}+\omega_{0}^{2}}
$$


where $G_{q}\left(n_{0}\right)$ is the road roughness coefficient and $\omega_{0}$ is the angular frequency $\left(\omega_{0}=0.011 \times 2 \pi v\right)$. (27):

The variance of $z_{10}$ can thus be determined using Eq.

$$
\sigma_{z_{10}}^{2}=2 \pi G_{q}\left(n_{0}\right) n_{0}^{2} v \cdot \int_{2 \pi v v_{1}}^{2 \pi v f_{2}}\left|\frac{H_{z 10-q}}{\omega_{0}+\mathrm{j} \omega}\right|^{2} \mathrm{~d} \omega
$$

where $f_{1}$ and $f_{2}$ are the lower and upper spatial frequency limits of the road respectively.

Since the suspension damping ratio is small:

$$
\sigma_{z_{10}}^{2} \approx 2 \pi G_{q}\left(n_{0}\right) n_{0}^{2} v \cdot \int_{0}^{+\infty}\left|\frac{H_{z_{10}-q_{0}}}{\omega_{0}+\mathrm{j} \omega}\right|^{2} \mathrm{~d} \omega
$$

Noting that:

$$
H_{\dot{z}_{10}-q_{0}}=\mathrm{j} \omega H_{z_{10}-q_{0}}
$$

Using James Formula $[21,22]$, the following can be obtained:

$$
\begin{gathered}
\sigma_{z_{10}}^{2}=\frac{\pi^{2} G_{q}\left(n_{0}\right) n_{0}^{2} v \cdot\left(M_{u}+M_{s}\right)}{c} \\
\sigma_{\dot{z}_{1}}^{2} \approx \frac{\pi^{2} G_{q}\left(n_{0}\right) n_{0}^{2} v \cdot k_{u}}{c}
\end{gathered}
$$

\subsection{Explicit expression for equivalent stiffness}

Substituting Eq. (33) into Eq. (14) gives:

$$
\frac{1.478 \alpha m_{z 1}}{h_{0}}-\frac{1.697 \alpha^{2}\left(m_{z_{1}}^{2}+\sigma_{z 10}^{2}\right)}{h_{0}^{2}}=0
$$

Substituting Eq. (35) and Eq. (25) into Eq. (19), $k_{\text {eq }}$, $m_{z 1}, \quad \sigma_{z 10}^{2}$ can be determined.

\section{Validation of Model using Numerical Simulation}

Using Eq. (29), filtered white noise is used to construct the road roughness time-domain excitation:

$$
\dot{q}(t)=-2 \pi n_{0} v \cdot q(t)+2 \pi n_{0} \sqrt{G_{q}\left(n_{0}\right) v} \cdot w(t)
$$

The suspension model parameters used in the simulation are listed in Table 1.

Table 1. Suspension and hydropneumatic spring arameters

\begin{tabular}{|c|c|}
\hline Parameters & Value \& Unit \\
\hline Gas polytropic exponent & 1.3 \\
\hline Sprung mass $M_{\mathrm{s}}$ & $7000 / \mathrm{kg}$ \\
\hline Unsprung mass $M_{\mathrm{u}}$ & $650 / \mathrm{kg}$ \\
\hline Stiffness of tire $k_{\mathrm{u}}$ & $1 \times 106 /(\mathrm{N} / \mathrm{m})$ \\
\hline $\begin{array}{c}\text { Gas chamber length at static } \\
\text { equilibrium position } h_{0}\end{array}$ & $96 / \mathrm{mm}$ \\
\hline Area of air chamber $A_{1}$ & $2.5 \times 104 / \mathrm{mm} 2$ \\
\hline Damping coefficient $c$ & $6 \times 104 /(\mathrm{Ns} / \mathrm{m})$ \\
\hline
\end{tabular}

\begin{tabular}{|c|c|}
\hline Area ratio $\alpha$ & 1.2 \\
\hline Road roughness coefficient $G_{q}\left(n_{0}\right)$ & $1024 \times 10-6 / \mathrm{m} 3$ \\
\hline Vehicle velocity $v$ & $15 /(\mathrm{m} / \mathrm{s})$ \\
\hline
\end{tabular}

Figure 3 shows the frequency domain input of the road roughness, It reveals that the time and frequency domain and distribution of the input is quite similar to what we want.

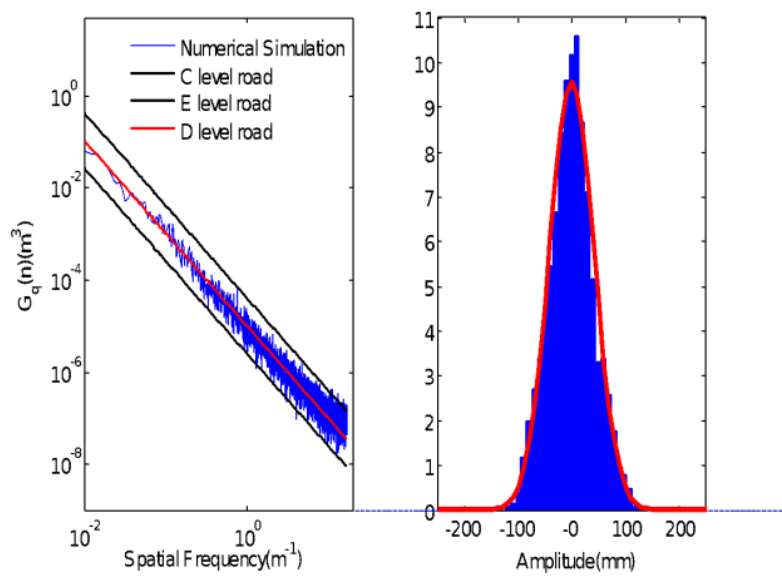

Figure 3. Validation of frequency and amplitude probability distribution

Figure 4 compares the hydropneumatic spring deformation determined through statistical linearization against that determined by the simulation and it shows that the distribution of the spring deformation closely approximates that of a Gaussian process.
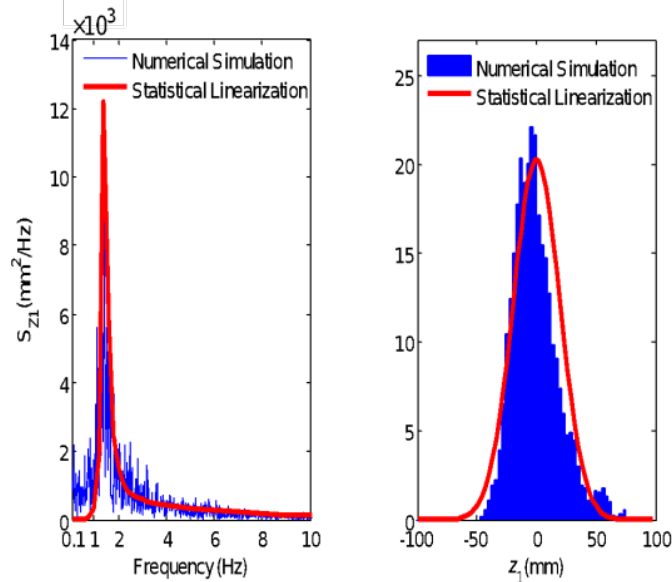

Figure 4. Hydropneumatic spring deformation distributions

Figure 5 compares the sprung mass acceleration determined through statistical linearization against that determined by the simulation and it shows that the results are of high accuracy in the frequency domain and the distribution. 

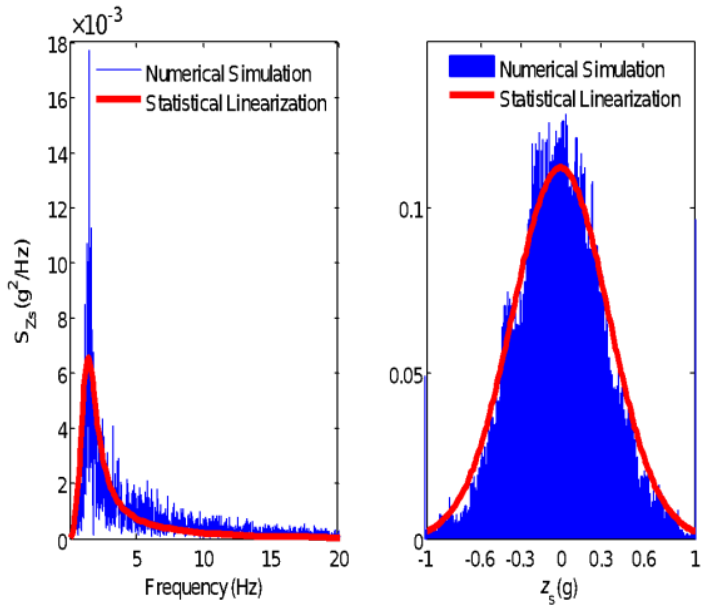

Figure 5. Sprung mass acceleration distributions

To sum up, the magnitude responses of the hydropneumatic spring deformation and the sprung mass acceleration determined through statistical linearization accurately approximate that determined by the simulation. The distributions of the hydropneumatic spring deformation and the sprung mass acceleration closely approximate that of a Gaussian process. Thus, the method of statistical linearization is validated.

To verify the response of the hydropneumatic suspension to different levels of road roughness and varying vehicle speed, two dimensionless parameters are introduced:

$$
y=\frac{\sigma_{z 1}}{h_{0}} \text { and } t=\frac{m_{z 1}}{h_{0}}
$$

where $y$ is the ratio of the hydropneumatic suspension deformation to the gas chamber length at the static equilibrium position and $t$ is the ratio of the dynamic mean-value offset to the gas chamber length at the static equilibrium position. The relation between the relative deformation and the vehicle speed and road roughness is revealed in Figure 6 .

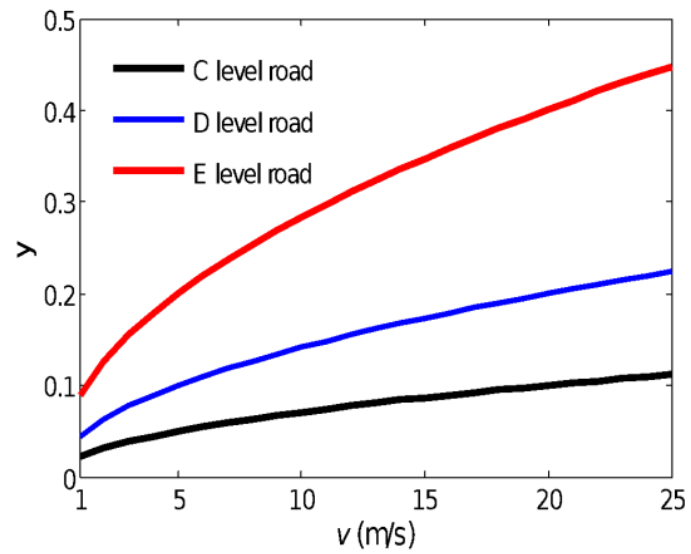

Figure 6. Relative deformation variation with vehicle velocity and road roughness

Figure 7 shows that, as the hydropneumatic suspension deformation increases, the dynamic meanvalue offset increases too.

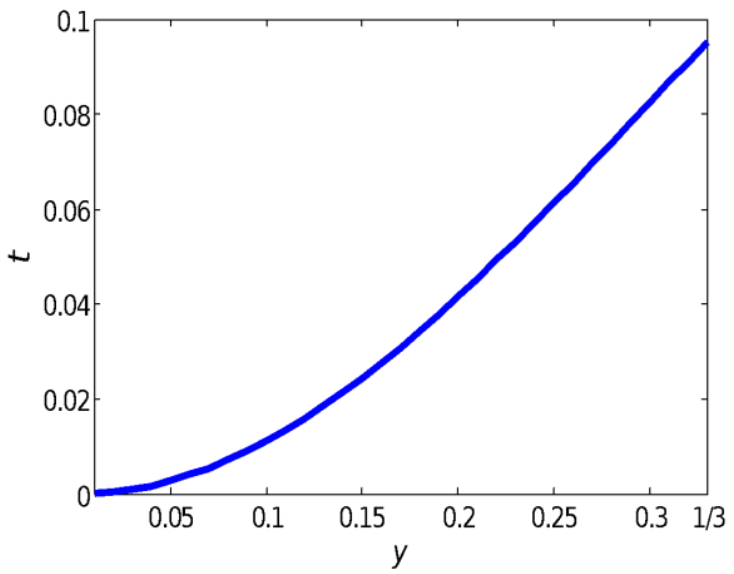

Figure 7. Relation of dynamic mean-value offset to hydropneumatic spring deformation

Figure 8 shows that, as vehicle speed and road roughness increase, the equivalent stiffness will increase as well. Thus, on a road in good condition, the small equivalent stiffness will provide a comfortable experience, but if the road is in a worse condition, the large equivalent stiffness will help the hydropneumatic suspension to absorb shock effectively.

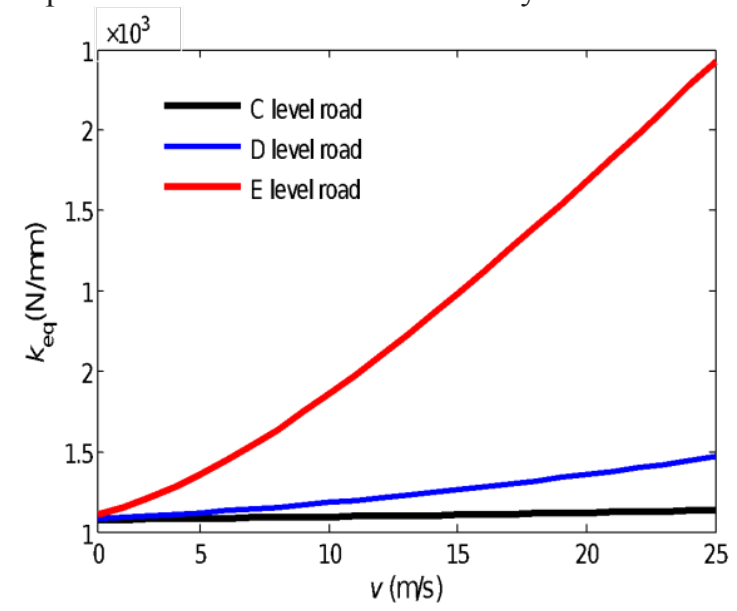

Figure 8. Equivalent stiffness with vehicle velocity and road roughness

This paper presents a novel method to depict nonlinear stiffness of hydropneumatic spring by approximating it with a quadratic polynomial. The results given in this paper can be further applied in advanced suspension system, i.e. active and semi-active suspension systems. Since the nonlinearities are affected by both road level and vehicle speed, it's believed that road adaptive advanced suspension system and adaptive observer will provide better performance with obtained results. For related topics, readers can refer to reference.

\section{Conclusions}

An accurate solution for the equivalent stiffness of a hydropneumatic suspension is obtained using the method of statistical linearization.A quadratic polynomial is used 
in computing and the results of the numerical simulation validate the accuracy of the statistical linearization method.Road roughness level and vehicle speed are considered in the analysis of the hydropneumatic suspension; as the vehicle speed and road roughness increase, the equivalent stiffness will increase too.

\section{References}

1. Solomon, U., and Padmanabhan, C., "Hydro-gas suspenseon system for a tracked vehicle: Modeling and analysis," Journal of Terramechanics, 2011, 48:125-137.

2. Konieczny, L., Burdzik, R., and Wegrzyn, T., "Analysis of structural and material aspects of selected elements of a hydro-pneumatic suspension system in a passenger car," Archives of metallurgy and materials, 2016, 61(1):79-83.

3. Daou, R.A.Z., Moreau, X., and Francis, C., "Effect of hydro-pneumatic components nonlinearities on the crone suspension," IEEE transactions on vehicular technology, 2012, 61(2): 466-474.

4. Ma, G., and Tan, R., "Nonlinear mathematic model of hydropenumatic suspension crane vehicles and its simulation," Chinese Journal of Mechanical Engineering, 2002, 38(5): 95-99.

5. Feng, S., "Research on nonlinear mathematical model and performance in hydro-pneumatic suspension," China Journal of Highway and Transport, 2002, 15(3): 122-126.

6. Zhu, W., "Random vibration," Beijing: Science Press, 1992:330-331. (in Chinese)

7. Emami, M. D., Mostafavi, S. A., and Asadollahzadeh, P., "Modeling and simulation of active hydropneumatic suspension system through bond graph," Mechanics, 2011, 17(3): 312-317.

8. Dong, M., and Luo, Z., "Statistical linearization on 2 DOFs hydro-pneumatic suspension with asymmetric nonlinear stiffness," Chinese Journal of Machanical Engineering, 2015, 28(3): 504-510.

9. Li, X., Shi, J., and Zhang, J., "Feedback linearization and PID control for active hydro-pneumatic suspension," Journal of ShangHai JiaoTong university, 2009, 43(10): 1521-1525.

10. Shi, J., Li, Xiaowei., and Zhang, J., "Feedback linearization control for missile launch vehicle active hydro-pneumatic suspension," Journal of System Simulation, 2009, 21(23): 7617-7622

11. Caughey, T. K., "Nonlinear theory of random vibration," Advances in Appl.Mech, 1977, 11: 209253.

12. Kazakov, I. E., "Generalization of the method of statistical linearization to multidimensional system,"
Automation and Remote Control, 1965a, 26: 12011206.

13. Iwan, W. D., "A Generalization of the method of equivalent linearization," International J. of Nonlinear Mech., 1973, 8: 279-283.

14. Atalik, T. S., "Stochastic linearization of multidegree-of-freedom non-linear systems," Earthquake Engineering and Structure Dynamics, 1976, 4: 411420.

15. Peng, J., Chen, S., and Chen H., "Equivalent linearization technique to a class of nonlinear multidegree of freedom system ," Journal of Vibration and Shock, 1995, (1): 30-35. (in Chinese)

16. Gai, Z., "Non-zero response for the hydro-pneumatic suspension in vehicles," Journal of Beijing Institute of Technology, 1990, 10(1): 8-13. (in Chinese)

17. Amirhossein, Y., Boris, L., "Order reduction of nonlinear hydropneumatic vehicle suspension," IEEE Inteinational Conference on Control Applications, 2006, 08(4): 1404-1408.

18. Boris, L., "Application of model order reduction to a hydropneumatic vehicle suspension," IEEE Transactions on Control System Technology, 1995, 3(1): 102-109.

19. Cao S., Yi, M., and Luo, X., "Statistic linearization analysis of the nonlinear stiffness model for hydropneumatic suspensions," Huazhong Univ. Of Sci. \& Tech. (Nature Science Edition), 2002, 30(6): 10-12. (in Chinese)

20. Roberts, J. B., "Response of nonlinear mechanical systems to random excitation: Part II; equivalent linearization and other methods," Shock and Vibration Digest, 1981, 13: 15-29.

21. James, H. M., Nichols, N. B., and Phillips, R. S., "Theory of Servomechanisms," New York: McGraw Hill, 1947.

22. Crandall, S. H., and Mark, W. D., "Random vibration in mechanical system," New York: Academic Press, 1963.

23. Wang, Z., Dong, M., Qin, Y., et al., "Suspension system state estimation using adaptive Kalman Filtering based on road classification," Vehicle System Dynamics, 2017, 55(3):371-398.

24. Qin, Y., Xiang, C., Wang, Z., and Dong, M., "Road Excitation Classification for Semi-Active Suspension System Based on System Response," Journal of Vibration and Control, (DOI: 10.1177/1077546317693432)

25. Qin, Y., Dong, M., Zhao, F., et al., "Road Profile Classification for Vehicle Semi-active Suspension System Based on Adaptive Neuro-Fuzzy Inference System," IEEE Control Decision Conference (CDC). Osaka, Japan. 2015, pp: 1533-1538. 